\title{
Flexible sweat sensors for non-invasive optimization of lithium dose in psychiatric disorders
}

\author{
Francesca Criscuolo ${ }^{* a}$, Filippo Cantù ${ }^{b}$, Irene Taurino $^{a}$, Sandro Carrara $^{a}$, Giovanni De Micheli ${ }^{a}$ \\ ${ }^{a}$ Swiss Federal Institute of Technology (EPFL), Lausanne, Switzerland \\ ${ }^{b}$ Department of Neurosciences and Mental Health, Fondazione IRCCS Ca' Granda Ospedale Maggiore Policlinico, \\ University of Milan, Milan, Italy \\ *Email: francesca.criscuolo@epfl.ch
}

\begin{abstract}
Lithium is the main drug in the prophylaxis of Bipolar Disorder (BD) as it acts as a mood stabilizer. However, due to its narrow therapeutic range, patients are obliged to frequent check-ups in hospital to control their lithium blood level and optimize the dose.

In this paper, we propose for the first time a wearable electrochemical sensor for the non-invasive decentralized Therapeutic Drug Monitoring (TDM) of lithium in sweat. The system is made of a platinum potentiometric sensor with an electrochemically nanostructured solid-contact and a silver Reference Electrode (RE). The optimal coverage and successful nanostructuration of the working electrode, useful to significantly reduce drift, is confirmed by Scanning Electron Microscopy (SEM). The integrated flexible RE is chemically chlorinated and covered with a PVC membrane doped with an Ionic Liquid (IL) to improve its stability. The sensing system is then tested both in aqueous solution and in sweat, showing quasi-Nernstian slope $(56.8 \pm 3.9$ $\mathrm{mV}$ /decade) in the range of clinical interest.
\end{abstract}

Index Terms-flexible sensors, wearable, electrochemistry, potentiometry, ion sensors, sweat.

\section{INTRODUCTION}

Lithium represents the most widely used mood stabilizer in psychiatric disorders [1]. Despite its narrow therapeutic window and possible severe side effects, it is still the main drug to treat people suffering from Bipolar Disorder (BD). In this paper, we present for the first time a wearable electrochemical sensor for the non-invasive decentralized Therapeutic Drug Monitoring (TDM) of lithium drug in sweat.

$\mathrm{BD}$ is a potentially mortal condition, which cannot be treated, but only controlled with specific drugs. It affects every year 60 million people worldwide (WHO) and $2.6 \%$ of the adult population in the U.S.A. (National Institute of Mental Health). It consists of recurring episodes of mania and depression. The risk of suicide is 30 times higher than for the general population, leading to a urgent need of mood stabilization with lithium drugs [2], [3]. The determination of the optimal lithium dose for each individual is a critical and difficult issue because of the narrow therapeutic range of this drug [4]. Poisoning can occur if the serum amount overcomes the maximum safe concentration, with severe consequences on patients' health, including myoclonic twitching, ataxia, drowsiness, chronic toxicity and irreversible damages to brain, liver, and kidneys [5]. It can eventually cause death. Interaction with other medicines and dietary restrictions also play an important role in the determination of the optimum dose [5].

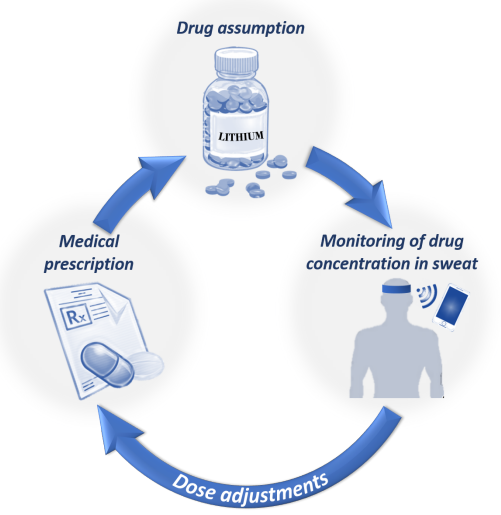

(a)

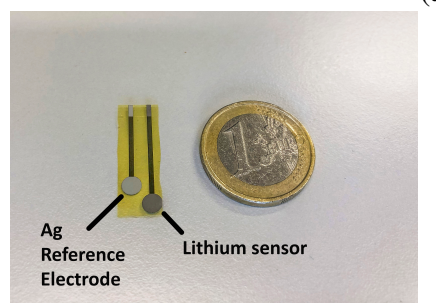

(b)

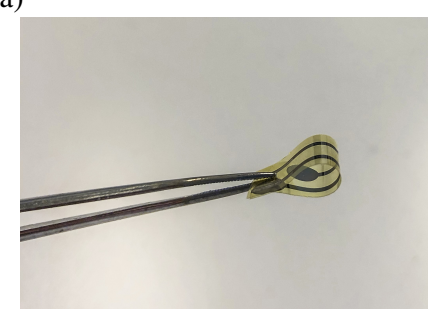

(c)
Fig. 1: (a) Scheme of the proposed non-invasive approach for Therapeutic Drug Monitoring (TDM) of lithium in sweat. (bc) The fabricated flexible electrochemical sensing platform for a wearable headband for lithium monitoring.

Consequently, TDM is a practice during lithium therapies [6], at the beginning of the treatment or after any change in the dose, by monitoring lithium concentration in serum at least every week (standardized $12 \mathrm{~h}$ or $24 \mathrm{~h} \mathrm{Li}^{+}$serum concentration). In the presence of some abnormalities the check-ups frequency should be increased. Only in absence of any complications it is possible to increase the intervals between subsequent analyses to about three months [1].

Clinical laboratories use complex and expensive techniques to quantify lithium, like atomic absorption spectrometry, flame emission photometry or conventional Ion-Selective Electrodes (ISEs) [7]. Electrochemical potentiometric sensors based on all-solid-state ISEs represent a promising alternative to substitute the complex and bulky traditional equipment thanks to their miniaturization, low cost and simplicity of measurements. 
Despite the realization of decentralized monitoring systems would significantly improve the life quality of the patients, only a few examples have been reported in literature in this direction so far [8], [9]. In addition, some major drawbacks should still be overcome, including the need of blood collection, toxicity, and reproducibility.

In this paper, we propose for the first time the use of a wearable headband for remote non-invasive monitoring of lithium levels in sweat. Chromatographic measurements have demonstrated the correlation between the amount of lithium extracted in sweat and the one present in serum [10]. In particular, the concentration in sweat is about 3 times higher than the one in blood (2.4 mM - 4.5 mM). Sweat offers several advantages over serum: it is readily accessible without the need of syringes and of invasive procedures, it is highly abundant and can be reproduced artificially following specific standards. A scheme of the proposed non-invasive approach for TDM of lithium in sweat is given in Figure 1a.

The microfabricated flexible electrochemical platform includes a nanostructured potentiometric sensor and a $\mathrm{Ag} / \mathrm{AgCl}$ all-solid-state Reference Electrode (RE) with a Polyvinyl Chloride (PVC) membrane doped with an Ionic Liquid (IL) to improve potential stability. The sensing system offers a quasiNernstian response $(56.8 \pm 3.9 \mathrm{mV} /$ decade) both in aqueous solution and in artificial sweat in the range of clinical interest.

\section{EXPERIMENTAL METHODS}

\section{A. Materials}

All chemicals were purchased from Sigma Aldrich (USA).

\section{B. Electrodes fabrication and functionalization}

The electrodes were fabricated with a lithography process as described in Fig. 2. Briefly, two lift-off processes were performed using a plasma-activated sputtering procedure for the deposition of silver and platinum. An adhesion promotion was performed before the deposition of the Polyimide (PI) passivation layer. Dry etching was then used for PI opening. Finally, the electrodes were released by anodic dissolution of the previously deposited aluminum layer in $1 \mathrm{M} \mathrm{NaCl}$ at 0.8 $\mathrm{V}$.

The platinum electrodes were cleaned by cyclic voltammetry in $0.5 \mathrm{M} \mathrm{H}_{2} \mathrm{SO}_{4}$ until complete overlapping of subsequent curves. Platinum nanostructures were then deposited on the working electrode by applying $-1 \mathrm{~V}$ in a $25 \mathrm{mM}$ $\mathrm{H}_{2} \mathrm{PtCl}_{6}, 50 \mathrm{mM} \mathrm{H} \mathrm{H}_{2} \mathrm{SO}_{4}$ aqueous solution using an $\mathrm{Au}-$ tolab PGSTAT $302 \mathrm{~N}$ potentiostat. A $10 \mu \mathrm{L}$ drop of IonSelective Membrane (ISM) cocktail ( 1 wt\% 6,6-dibenzyl1,4,8-11-tetraoxacyclotetradecane, $28.00 \%$ PVC high molecular weight, $70.3 \mathrm{wt} \% 2$-nitrophenyl octyl ether and $0.7 \mathrm{wt} \%$, potassium tetrakis (4-chlorophenyl)borate dissolved in THF ) was deposited on the electrodes.

The flexible $\mathrm{Ag} \mathrm{RE}$ was chlorinated in $50 \mathrm{mM} \mathrm{FeCl}_{3}$ before membrane deposition. The RE membrane cocktail contains 0.1 $\%$ of IL (1-dodecyl-3-methylimidazolium chloride), $33 \%$ of PVC powder, $66 \%$ of Bis(2-ethylhexyl) sebacate in $0.5 \mathrm{~mL}$ of THF. A volume of $10 \mu \mathrm{L}$ was drop-cast onto the electrodes.

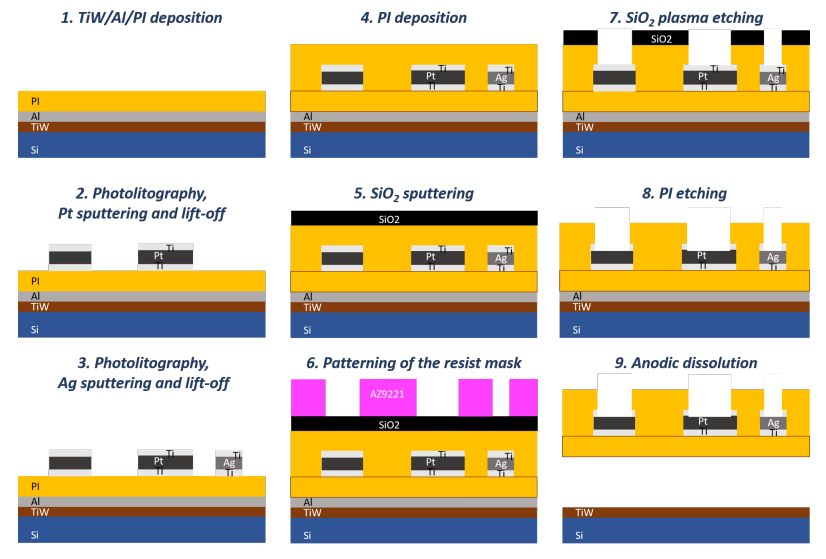

Fig. 2: Fabrication steps of the flexible electrochemical $\mathrm{Li}^{+}$sensing platform on a PI substrate.

The solvent was allowed to evaporate overnight. The ISEs were conditioned in $10^{-2} \mathrm{M} \mathrm{LiCl}$ for 24 hours.

\section{Morphological and electrochemical characterization}

The Scanning Electron Microscopy (SEM) analysis was performed with a Gemini 300 microscope from Zeiss at the Interdisciplinary Centre of Electronic Microscopy (CIME) of EPFL in SE mode. The potentiometric measurements were performed in a two-electrodes setup using an EMF6 precision electrode interface by Lawson lab. The artificial sweat was prepared as described in [11].

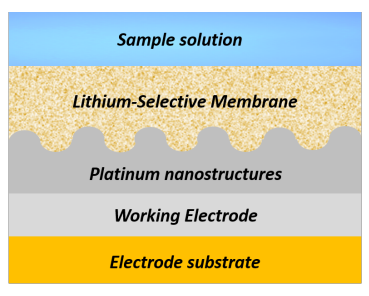

(a)

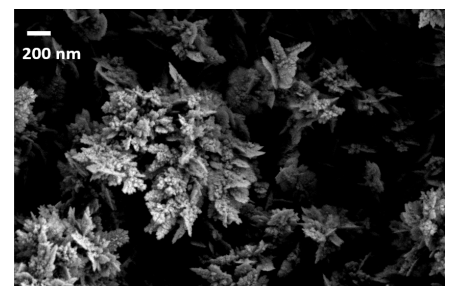

(b)
Fig. 3: (a) Structure of the $\mathrm{Li}^{+}-$ISE based on platinum nanostructured solid-contacts. (b) SEM image of the platinum nanoflowers deposited on the flexible sensing electrodes.

\section{EXPERIMENTAL RESULTS AND DISCUSSION}

In this paper, we fabricate and test for the first time a complete flexible electrochemical sensing platform for noninvasive monitoring of lithium in perspiration (Fig. 1b). A platinum working electrode and a silver RE are patterned on a PI substrate using lithography techniques. The complete flexibility of the final system is evident in Figure 1c.

The structure of the sensing electrodes is reported in Figure 3a. The nanostructuration of the solid-contacts has been demonstrated to be an efficient way to improve potential response and stability. In particular, in our previous work we have proved the superior behaviour of platinum nanoflowers as solid-contacts on rigid screen-printed electrodes [12]. 


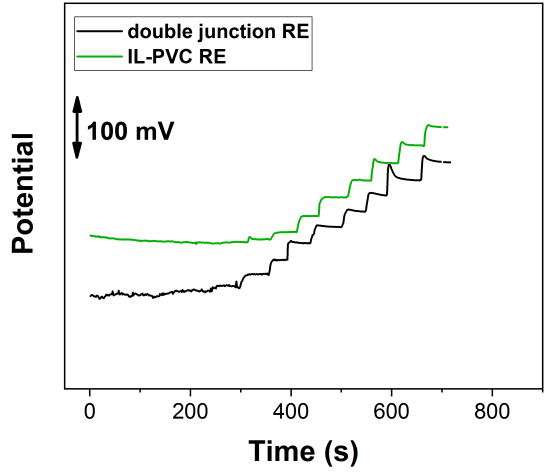

Fig. 4: Calibration time traces obtained with the flexible RE and with a $\mathrm{Ag} / \mathrm{AgCl}$ double junction $\mathrm{RE}$.

TABLE I: Analytical parameters of the $\mathrm{Li}^{+}$-sensing system in water and in sweat.

\begin{tabular}{|cl|c|c|}
\hline & & $\begin{array}{c}\text { sensitivity } \\
{[\mathrm{mV} / \text { decade }]}\end{array}$ & $\begin{array}{c}\text { LOD } \\
(\mathrm{M})\end{array}$ \\
\hline \multirow{2}{*}{ WATER } & This work (flexible) & $\mathbf{5 9 . 6} \pm 1.5$ & $(\mathbf{5 . 9} \pm 2.6) \times 10^{-5}$ \\
& Literature (rigid) & $\mathbf{5 8 . 7} \pm 0.8$ & $(\mathbf{1 . 3} \pm 0.4) \times 10^{-5}$ \\
\hline \multirow{2}{*}{ SWEAT } & This work (flexible) & $\mathbf{5 6 . 8} \pm 3.9$ & $(\mathbf{1 . 7} \pm 0.6) \times 10^{-3}$ \\
& Literature (rigid) & $\mathbf{5 7 . 6} \pm 2.1$ & $(\mathbf{1 . 4} \pm 0.2) \times 10^{-3}$ \\
\hline
\end{tabular}

The electrode nanostructuration required an accurate cleaning procedure before the electrodeposition to ensure a uniform and controlled process with maximization of the surface area. The electrochemical cleaning in $\mathrm{H}_{2} \mathrm{SO}_{4}$ by $\mathrm{CV}$ was found to be the best way to achieve high surface roughness, coverage and reproducibility. A one-step potentiostatic electrodeposition process is used. The efficiency of the nanoflowers deposition was proved by the similar roughness factors $(\sim 200)$ with respect to the one on rigid substrate on screen-printed electrodes, obtained as described in [13]. Morphological analysis by SEM further corroborates these results (Fig. 3b).

A stable RE is crucial to ensure accurate measurements, especially in potentiometric sensors where the precision on potential has to be optimal. A chemical chlorination was used as the electrochemical one was found to give similar results, while requiring a more laborious procedure. The produced $\mathrm{Ag} / \mathrm{AgCl} \mathrm{RE}$ was then covered with an IL-doped PVC to improve potential stability. The calibration time trace of the RE (obtained during half-log increases of the concentration) was compared to the one obtained with a conventional $\mathrm{Ag} / \mathrm{AgCl}$ double junction RE (Fig. 4) to prove its efficiency. The flexible RE shows very limited drift at low concentrations and almost identical steps height in the range of interest.

Finally the complete system was tested both in aqueous solution and in sweat (Fig. 5). The corresponding sensor parameters are reported in Table I in comparison with the literature values for rigid systems. In both cases, a quasiNernstian response $(56.8 \pm 3.9 \mathrm{mV} /$ decade $)$ is achieved in the concentration range of clinical interest. Both selectivities and

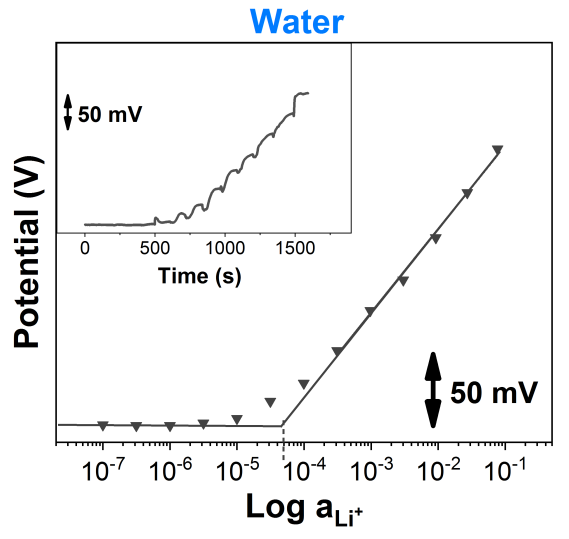

(a)

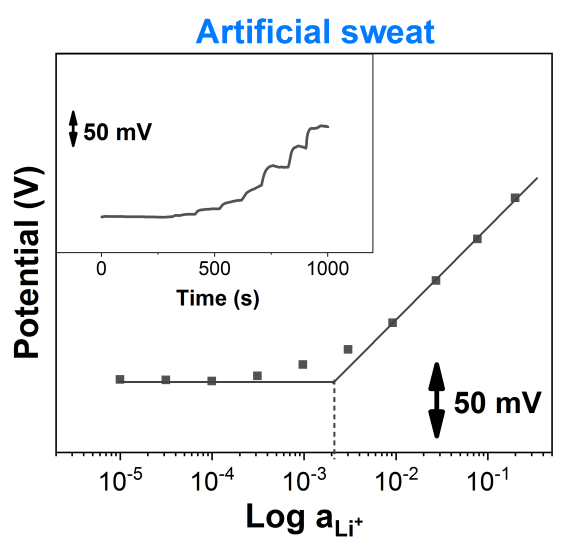

(b)

Fig. 5: $\mathrm{Li}^{+}$calibrations obtained with the flexible electrochemical sensing platform in water (a) and in artificial sweat (b) by subsequent $\mathrm{LiCl}$ additions. The flexible all-solid-state $\mathrm{RE}$ is used in all measurements.

LOD values are comparable to the one reported in literature for rigid sensors. Further improvements could be obtained by proper tuning of the conditioning procedure.

\section{CONClusion}

A complete flexible electrochemical sensing platform for non-invasive monitoring of lithium levels is proposed in this work. The efficient nanostructuration is proved by the high roughness factor $(\sim 200)$ and by SEM analysis. The stability of the integrated all-solid-state RE is demonstrated by comparison with a $\mathrm{Ag} / \mathrm{AgCl}$ double junction $\mathrm{RE}$. The complete system is then tested for lithium calibration both in water and in sweat. A quasi-Nernstian response $(56.8 \pm 3.9 \mathrm{mV} /$ decade) is obtained in both cases in the range of clinical interest $\left(\mathrm{LOD}=(1.7 \pm 0.6) \times 10^{-3}\right)$.

\section{ACKNOWLEDGMENT}

This research is supported by H2020 ERC 2014 ADG669354 CyberCare. 


\section{REFERENCES}

[1] R. W. Licht, "Lithium : Still a Major Option in the Management of Bipolar Disorder Acute Antimanic Actions of Lithium," CNS Neurosci. Ther, vol. 18, pp. 219-226, 2012.

[2] M. Pompili, M. Innamorati, M. Raja, G. Ducci, G. Angeletti, D. Lester, P. Girardi, R. Tatarelli, and E. D. Pisa, "Suicide risk in depression and bipolar disorder : Do impulsiveness-aggressiveness and pharmacotherapy predict suicidal intent?" Neuropsychiatr. Dis. Treat., vol. 4, no. 1, pp. 247-255, 2008

[3] A. Muneer, "Staging Models in Bipolar Disorder : A Systematic Review of the Literature," Clin. Psychopharmacol. Neurosci., vol. 14, no. 2, pp. $117-130,2016$.

[4] D. Gruson, A. Lallali, A. M. Taburet, A. Legrand, M. Conti, and L. D. Biochimie, "Evaluation of a new lithium colorimetric assay performed on the Dade Behring Dimension [U+1B8B] X-pand [U+0BC0] system," Clin. Chem. Lab. Med., vol. 42, no. 9, pp. 1066-1068, 2004.

[5] H. S. Hopkins and J. Gelenberg, "Serum lithium levels and the outcome of maintenance therapy of bipolar disorder," Bipolar disord., vol. 2, pp. $174-179,2000$

[6] G. D. Christian, "Reagents for Lithium Electrodes and Sensors for Blood Serum," Sensors, vol. 2, pp. 432-435, 2002.

[7] B. Rumbelow and M. Peake, "Performance of a novel spectrophotometric lithium assay on a routine biochemistry analyser," Ann. Clin. Biochem., vol. 38, pp. 684-686, 2001.

[8] M. Novell, T. Guinovart, P. Blondeau, F. X. Rius, and F. J. Andrade, "A paper-based potentiometric cell for decentralized monitoring of $\mathrm{Li}$ levels in whole blood," Lab Chip, vol. 14, no. 7, p. 1308, 2014.

[9] F. Coldur and M. Andac, "All-Solid-State Polyvinyl Chloride Membrane Lithium-Selective Electrode with Improved Selectivity and Its Application in Serum Lithium Assay," Sensor Lett., vol. 9, no. 5, pp. 1738-1744, 2011.

[10] B. Leboulanger, J. M. Aubry, G. Bondolfi, R. H. Guy, and M. B. Delgado-Charro, "Lithium monitoring by reverse iontophoresis in vivo," Clinical Chemistry, vol. 50, no. 11, pp. 2091-2100, 2004

[11] T. Kilic, V. Brunner, L. Audoly, and S. Carrara, "Smart e-Patch For Drugs Monitoring in Schizophrenia," in 2016 IEEE International Conference on Electronics, Circuits and Systems (ICECS). IEEE, 2016, pp. $57-60$.

[12] F. Criscuolo, I. Taurino, F. Stradolini, S. Carrara, and G. De Micheli, "Highly-stable $\mathrm{Li}+$ ion-selective electrode based on noble metal nanostructures as solid-contacts," Anal. Chim. Acta, vol. 1027, pp. 22-32, 2018.

[13] F. Criscuolo, I. Taurino, V. A. Dam, and F. Catthoor, "Fast Procedures for the Electrodeposition of Platinum Nanostructures on Miniaturized Electrodes for," Sensors, vol. 19, no. 2260, pp. 1-12, 2019. 